\section{Diagnostische Untiefen umschiffen!}

\author{
Die Diagnostik bei Überempfindlichkeitsreaktionen auf Arzneimittel \\ war ein wichtiges Thema bei der diesjährigen Herbsttagung der \\ Regionalgruppe Nord des Ärzteverbandes Deutscher Allergologen. \\ Anamnese und Hauttestungen sind dabei wichtige Instrumente.
}

D ie Diagnose von Arzneimittelüberempfindlichkeitsreaktionen ist schwierig, gab Prof. Dr. Bettina Wedi, Hannover, zu: Zur Variabilität des klinischen Bildes komme meist ein unklarer Pathomechanismus, relevante Epitope blieben oft unbekannt. Zusätzlich seien vorhandene In-vivo- und In-vitro-Tests oftmals nicht standardisiert, so Wedi.

\section{Ausgangspunkt: Anamnese}

Bei der Abklärung steht die Anamnese an erster Stelle. Dabei sollte vor allem auf Arzneimittel fokussiert werden, die in den letzen zwei bis vier Wochen gegeben worden sind und die zunächst über einige Tage gut vertragen wurden. Des Weiteren kann aufgrund von Erfahrungswerten eine immunologische von einer eher nicht immunologischen Reaktion unterschieden werden. Klassische IgEvermittelte Reaktionen werden z. B. durch $\beta$-Laktamantibiotika, Fluorchinolone oder Pyrazole hervorgerufen. Die Reaktionen auf NSAR, Kontrastmittel und Lokalanästhetika werden dagegen eher durch nicht immunologische Mechanismen hervorgerufen. Das klinische Bild gleicht sich allerdings häufig.

Bei der Untersuchung der Hautsymptome sind urtikarielle, makulopapulöse, bullöse und pustulöse Primärläsionen meist auf ein einzelnes Arzneimittel zurückzuführen. Schwieriger wird die Differenzialdiagnose bei einer thrombozytopenischen Purpura oder einer allergischen Vaskulitis, da nicht nur Arzneimittel, sondern auch infektiöse Erreger das Krankheitsbild auslösen können. Werden dann für die Infekttherapie Antibiotika und NSAR verordnet und es entstehen Hautreaktionen, wird die Ursachensuche nicht ganz einfach. Grundsätzlich sollte daher immer nach Co-Faktoren einer Arzneimittelreaktion gefragt werden. Dazu ge- hören Infekte, körperliche Anstrengung, auch UV-Strahlung oder bestimmte Nahrungsmittel.

\section{Begrenzte Aussagekraft: Haut- und In-vitro-Testungen}

In der niedergelassenen Praxis wird man in der Regel einen Hauttest zur diagnostischen Abklärung durchführen. Dies sollte nicht während der aktuellen Symptomatik geschehen, der beste Zeitpunkt ist sechs Wochen bis sechs Monate nach der Reaktion. Die Testungen sollten möglichst mit dem Originalpräparat erfolgen,

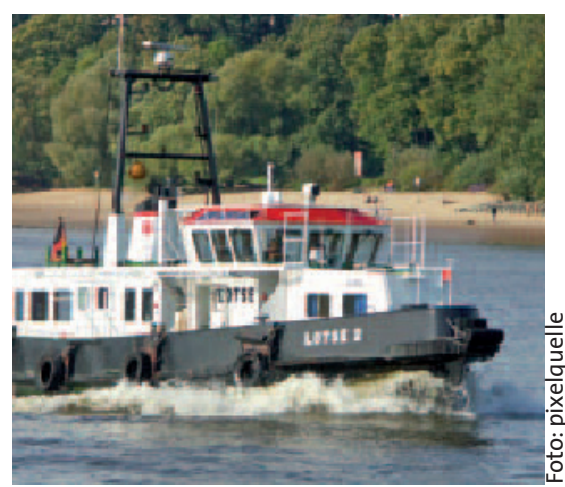

Bei der Herbsttagung der ÄDA-Regionalgruppe Nord am 4. November 2006 in Hamburg lotsten die Referenten das Auditorium sicher durch das diagnostische Vorgehen bei Arzneimittelreaktionen.

zusätzlich mit dem reinen Wirkstoff sowie galenischen Hilfsstoffen wie etwa dem Tablettensprengmittel. Der Pricktest wird in der Regel mit unverdünntem Material durchgeführt, der Intrakutantest in Verdünnungen $\mathrm{ab} 10^{-4}$ bis $10^{-1}$ in physiologischer Kochsalzlösung und nur steril. Bei den Epikutantestungen eignen sich je nach Arzneistoff Vaseline, Wasser oder Alkohol als Trägermaterial.

Vorsicht bei den Hauttests ist z. B. bei akuter generalisierter exanthema- tischer Pustulose, Stevens-Johnson-Syndrom oder toxischer epidermaler Nekrolyse geboten. Auch bei Tests mit den Arzneimitteln Aciclovir, Carbamazepin und Pseudoephedrin sollte überlegt vorgegangen werden. Als Kontraindikationen gelten Schwangerschaft und nicht sicher medikamentös beherrschbare Überempfindlichkeitsreaktionen wie schweres Asthma. Generell gilt aber: Ein negativer Hauttest schließt eine Medikamentenüberempfindlichkeit nicht aus und umgekehrt bestätigt ein positiver Hauttest nicht unbedingt eine Medikamentenüberempfindlichkeit.

Bei Unsicherheiten im Hauttest oder bei Kontraindikationen kann bei einigen ausgewählten Arzneimitteln recht gut eine In-vitro-Testung weiterhelfen, erklärte Dr. Hagen Ott, Aachen. Kommerziell angeboten werden Fluoreszenz-Enzym-Immunoassays für verschiedene $\beta$ Laktame. Der optimale Testzeitpunkt ist bis maximal sechs Monate nach der Reaktion. Allerdings sind die Testungen recht teuer und noch liegen keine definierten Cut-off-Werte vor. Noch im Experimentalstadium sei die Basophilenaktivierung, sagte Ott. Es liegen nur wenige Studien mit mehr als 20 Patienten vor, außerdem sind die Testungen nur wenig standardisiert. Das gleiche gilt für den Lymphozytentransformationstest.

\section{Goldstandard: Provokationstestungen}

Die Provokationstestung als Goldstandard und einzig (relativ) sichere Methode in der Diagnostik birgt Risiken und sollte unter standardisierten Bedingungen stattfinden. Im Prinzip sei es eine Methode, die in die Klinik gehöre, warnte Dr. Michael Körner, Hannover - einzige Ausnahme: Die Testung bei Verdacht auf Lokalanästhetika-Unverträglichkeit könne auch in der niedergelassenen Praxis gefahrlos durchgeführt werden, die Provokation bestehe hier in einer subkutanen Applikation des verdächtigen Stoffs. Zur Standardisierung gehören eine Testung in einem infektfreien Intervall und ein genügend großer Abstand zu z. B. Rotweingenuss am Abend, zusätzlich muss natürlich immer eine Plazebokontrolle erfolgen. Auf keinen Fall sollten Provokationstestungen eingeleitet werden, wenn lediglich Patientenhinweise ohne gesicherte diagnostische Daten vorliegen. 